\title{
Fatores que predispõem ao declínio funcional em idosos hospitalizados
}

\author{
Predictors of functional dedine in hospitalized elderly patients
}

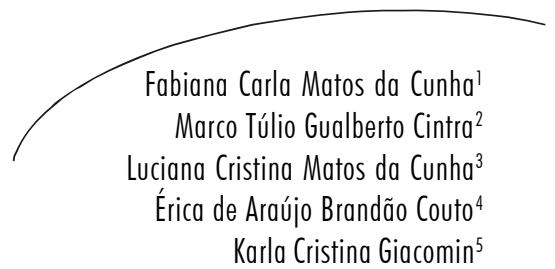

\section{Resumo}

Introdução: $\mathrm{O}$ aumento da expectativa de vida é uma realidade mundial e determina maior prevalência de doenças crônicas e maior demanda pelos serviços de saúde, incluindo os hospitalares. Atualmente, estudos têm focalizado o declínio funcional e suas consequências - demanda por recursos materiais e humanos, institucionalização e hospitalização. O objetivo foi a revisão crítica da literatura disponível sobre os fatores de risco associados ao declínio funcional em idosos hospitalizados. Metodologia: Pesquisa nas bases de dados MEDLINE, LILACS e SciELO, nos idiomas inglês e português, entre 2000 e 2007, utilizando as palavras-chave hospital, elderly, functional status, aged e functional decline e referências bibliográficas dos artigos selecionados. Resultados: Satisfizeram os critérios de inclusão 31 publicações. Após leitura crítica do abstract, os 15 artigos mais relevantes foram selecionados. Conclusão: O declínio funcional pode ocorrer antes, durante e/ou após a hospitalização. Idade avançada, comprometimento funcional prévio, déficit cognitivo, iatrogenias, gravidade da condição clínica e histórico de quedas foram os fatores de risco mais citados. A capacidade funcional, como marcador de saúde desse grupo, merece maior investigação.

1 Hospital São Judas Tadeu, Prefeitura Municipal de Ribeirão das Neves. Ribeirão das Neves, MG, Brasil

2 Universidade Federal de Minas Gerais, Programa de Pós-Graduação em Ciências da Saúde. Belo Horizonte, MG, Brasil.

3 Universidade Federal de Minas Gerais, Hospital das Clínicas, Residência médica em Geriatria. Belo Horizonte, MG, Brasil

4 Universidade Federal de Minas Gerais, Faculdade de Medicina, Departamento de Oftalmologia e Otorrinolaringologia. Belo Horizonte, MG, Brasil

5 Universidade Federal de Minas Gerais, Escola de Educação Física, Departamento de Terapia Ocupacional, Curso de Pós-graduação em Gerontologia. Belo Horizonte, MG, Brasil

Correspondência / Correspondence

Fabiana Carla Matos da Cunha

E-mail: fabyanacarla@bol.com.br
Palavras-chave:

Idoso.

Hospitalização.

Fatores de Risco.

Saúde do Idoso.

Funcionalidade 


\section{Abstract}

Introduction: The increase in life expectancy is a global reality and determines the highest prevalence of chronic diseases and greater demand for health services, including hospitals. Currently, studies have focused on functional decline and its consequences - the demand for material and human resources, institutionalization and hospitalization. This paper aimed to critically review the available literature on the risk factors associated with functional decline in hospitalized elderly people. Methodology: Search the databases MEDLINE,

Key words: Elderly. Functioning. Hospitalization. Risk Factors. Health of the Elderly. LILACS and SciELO, in English and Portuguese between 2000 and 2007, using the key words hospital, elderly, functional status, aged and functional decline and references of selected articles.

Results: Thirty-one publications met the inclusion criteria. After critical reading of the abstract, the 15 most relevant papers were selected. Conclusion: The functional decline can occur before, during and / or after hospitalization. Advanced age, prior functional impairment, cognitive impairment, iatrogenic, severe medical condition and history of falls were the risk factors most often cited. Functional capacity, as a marker of health in this group, deserves further investigation.

\section{INTRODUÇÃO}

O acelerado processo de envelhecimento populacional é observado tanto nos países desenvolvidos quanto naqueles em desenvolvimento, como é o caso do Brasil. A velocidade em que acontece esse envelhecimento se tornou um tema da atualidade, sobretudo quando a discussão atinge a questão do (des)preparo do sistema de saúde para acolher as demandas dessa parcela crescente da população. ${ }^{1}$

No Brasil, a partir de 1940, vem sendo observada uma redução significativa do número de crianças e adolescentes em relação ao total da população, e aumento da morbi-mortalidade por doenças crônico-degenerativas e por causas externas nas faixas etárias mais avançadas. ${ }^{2,3} \mathrm{~A}$ evolução dessas doenças é frequentemente marcada pela redução progressiva da capacidade funcional, com consequente comprometimento da qualidade de vida. ${ }^{2,4}$

Em 1980, a Organização Mundial de Saúde ${ }^{5}$ publicou a classificação Internacional de Deficiências, Incapacidades e Desvantagens (CIDID), que definiu a incapacidade funcional como a dificuldade na realização de atividades típicas e pessoalmente desejadas na sociedade, devido a uma deficiência orgânica. ${ }^{6}$

O processo de revisão da CIDID culminou com a aprovação, pela Assembleia Mundial da Saúde, em 2001, da Classificação Internacional de Funcionalidade, Incapacidade e Saúde (CIF). Esse modelo de classificação fornece a descrição da saúde e dos 
estados relacionados à mesma, além de permitir uma linguagem padronizada entre os profissionais. Segundo ele, a incapacidade funcional resulta não apenas de uma deficiência orgânica, mas da interação entre a disfunção apresentada pelo indivíduo, à limitação de suas atividades, à restrição da participação social e aos fatores ambientais e pessoais que interferem no seu desempenho em AVDs, podendo funcionar como barreiras ou facilitadores do estado funcional. ${ }^{5,6}$

Na parcela idosa da população, a redução gradual e progressiva da capacidade funcional, o processo de envelhecimento, bem como a carga de afecções crônicas resultam em maior consumo de serviços de saúde, tanto ambulatoriais quanto hospitalares. Os idosos apresentam taxas de internação hospitalar bem mais elevadas do que as observadas em outros grupos etários, assim como uma permanência hospitalar mais prolongada e uma recuperação mais lenta e complicada. ${ }^{47}$ As hospitalizações podem resultar em repercussões na capacidade funcional e em mudanças na qualidade de vida, muitas vezes de forma irreversível. ${ }^{8,9}$

Os fatores de risco para o declínio funcional no idoso hospitalizado ainda são pouco conhecidos e, por isso, têm sido investigados por vários estudos. Estes estudos têm discutido se a atuação sobre estes fatores teria impacto sobre a mortalidade, tempo de hospitalização e morbidade, incluindo a capacidade funcional e a qualidade de vida. ${ }^{9-12}$

A identificação precoce dos fatores de risco para o declínio funcional em idosos hospitalizados pode fornecer subsídios à equipe de assistência hospitalar quanto à adequação de protocolos de avaliação, o direcionamento das intervenções e à identificação de grupos que se beneficiariam de intervenções geriátricas específicas, medidas que contribuiriam para a eficiência e qualidade dos serviços prestados. ${ }^{7,13}$

Em virtude da extrema importância do tema, o objetivo do presente estudo foi a revisão crítica da literatura disponível sobre o declínio funcional associado à hospitalização em idosos e discutir os fatores a ele associados.

\section{METODOLOGIA}

Foram elegíveis artigos de estudos observacionais/longitudinais, publicados nos idiomas inglês e português entre os anos de 2000 e 2007, realizados na população idosa em ambiente hospitalar, que contivessem as palavras-chave selecionadas (bospital elderly AND functional status; elderly AND hospitalization AND functional; bospitalized aged AND functional decline e os termos equivalentes em português).

Duas estratégias foram utilizadas para pesquisar os artigos relevantes para esta revisão. A primeira consistiu de uma busca bibliográfica nas bases de dados MEDLINE, LILACS e SciELO, compreendendo o período de janeiro de 2000 a julho de 2007. A segunda consistiu na identificação de outros estudos relevantes a partir da revisão das referências bibliográficas de todos os artigos inicialmente selecionados. Con- 
sideradas as duas estratégias, foi encontrado um universo de 93 artigos, dos quais 31 preencheram os critérios de inclusão. Foram excluídos os trabalhos realizados na comunidade, os ensaios clínicos randomizados e as pesquisas não relacionadas à capacidade funcional.

Após a leitura dos resumos, foram selecionados 15 artigos de maior relevância, que foram lidos na íntegra e analisados quanto aos objetivos, tipo de estudo, características da amostra, medidas utilizadas e preditores significativos identificados.

\section{RESULTADOS}

Do ponto de vista metodológico, observou-se que oito estudos utilizaram desenho de coorte longitudinal prospectivo; ${ }^{13-19}$ três estudos foram do tipo observacional longitudinal; ${ }^{20-22} \mathrm{um}$, do tipo descritivo exploratório ${ }^{23} \mathrm{e}$ três coortes selecionadas a partir de ensaios clínicos. ${ }^{2426}$ Todos utilizaram análises estatísticas descritivas de variáveis clínicas e sócio-demográficas.

Houve grande variação quanto a: critérios de exclusão e de inclusão utilizados nos estudos, número de idosos incluídos nas amostras, características sócio-demográficas, bem como o tempo de seguimento dos mesmos, a duração da internação hospitalar, o momento da avaliação funcional e as escalas utilizadas.

Os critérios de exclusão mais utilizados pelos estudos foram: idosos com tempo de hospitalização menor do que $48 \mathrm{~h}$, que foram transferidos para outros hospitais ou para unidades de terapia intensiva, que evoluíram a óbito durante a hospitalização ou que apresentavam dependência total em AVDs à admissão. Apenas cinco estudos incluíram idosos institucionalizados. . $^{15,17,22,26,27}$

O menor estudo investigou 28 idosos $^{23}$ e o maior incluiu 2.877 idosos em um período de seguimento que foi desde a admissão hospitalar até um ano após a alta. ${ }^{17}$

No que se refere às características da amostra, observou-se, na maioria dos estudos, que os participantes eram mulheres, viúvas, brancas, com alguma dependência em AVDs. ${ }^{13-17,20-27}$ A média de idade dos participantes dos estudos estrangeiros foi acima de 80 anos e dos dois estudos em português foi de 68 e 78 anos, respectivamente. ${ }^{21,23}$

Quanto ao tempo de seguimento dos estudos, o maior foi de cinco anos ${ }^{13}$ e o menor entre a admissão hospitalar e o quarto dia de hospitalização. ${ }^{18}$

O tempo médio de internação foi semelhante entre os estudos, sendo de aproximadamente 15 dias, apesar das variações (3 a 79 dias). As principais causas de hospitalização apontadas por todos os estudos analisados foram: doenças cardiovasculares, cerebrovasculares, problemas pulmonares e gastro-intestinais.

Em relação à capacidade funcional, verificou-se que esta foi avaliada com frequência à admissão hospitalar, retrospectivamente 
no período anterior à admissão, durante o período de hospitalização e no momento da alta. ${ }^{15,16,21,22,24,26}$ Para sua avaliação, os instrumentos mais utilizados pelos estudos em ordem descrescente foram: medidas não-sistematizadas sobre AVDs, ${ }^{15,16,19,22,24,26,27}$ indice de Katz, ${ }^{13,17,20,26}$ escala de Lawton, ${ }^{13,14}$ OMFAQ/ BOMFAQ - versão brasileira, ${ }^{5,10}$ Medida de Independência Funcional $\mathrm{MIF}^{23} \mathrm{e}$ Indicador de BARTHEL. ${ }^{14}$

Em relação à avaliação das funções cognitivas e humor, observou-se que os testes mais utilizados pelos estudos foram o Miniexame do Estado Mental (MEEM), ${ }^{13,15,16,18,21}$ Short Portable Mental Status Questionnaire (SPMSQ) $)^{14,17,20,24-26}$ e a Escala de Depressão Geriátrica (GDS). ${ }^{14,18,22} \mathrm{Em}$ menor frequência, os estudos realizaram avaliações por meio do Confusion Assesment Method (CAM), ${ }^{18,21}$ NEECHAM scale, ${ }^{18,19}$ Hodkinson Abreviat Mental status, ${ }^{22}$ uma pergunta para avaliar depressão/humor, ${ }^{13} \mathrm{e}$ a Escala Modificada de Blessed para Classificação da Demência. ${ }^{16}$

Quanto aos aspectos clínicos para avaliação e classificação do número e gravidade das comorbidades, foram utilizados: Charlson Comorbidity Index, Acute Physiology And Chronic Health Evaluation (APACHE II scale), exames laboratoriais, Cumulative Illness Rating Scale (CIRS), Acute Physiology and Chronic Health Evaluation (APS) e a miniavaliação nutricional.

Em relação aos fatores predisponentes ao declínio funcional em idosos durante o período de hospitalização, verificou-se que o comprometimento funcional prévio à admissão; a presença de déficit cognitivo, incluindo estado confusional agudo; a gravidade da condição clínica (diagnóstico de acidente vascular encefálico, múltiplas comorbidades e entrada pelo pronto-socorro); idade avançada; mobilidade reduzida, incluindo repouso prolongado no leito e instabilidade postural e o histórico prévio de quedas foram os mais apontados pelos estudos aqui avaliados. Em menor frequência, também foram citados depressão, baixo índice de massa corpórea e de outros marcadores nutricionais, polifarmácia, autopercepção de saúde ruim, fatores ambientais relacionados à assistência fornecida, incontinência urinária e institucionalização.

Quadro 1 - Fatores de risco para o declínio funcional em idosos hospitalizados. 2007.

\begin{tabular}{|c|c|}
\hline $\begin{array}{l}\text { Fatores Sócio - } \\
\text { demográficos }\end{array}$ & Idade avançada e institucionalização \\
\hline $\begin{array}{l}\text { Fatores } \\
\text { Médicos/Clínicos }\end{array}$ & $\begin{array}{l}\text { Diagnóstico de AVE*, múltiplas comorbidades, gravidade da condição } \\
\text { clínica e estado nutricional } \\
* \text { AVE = Acidente Vascular Encefálico }\end{array}$ \\
\hline $\begin{array}{l}\text { Fatores Relacionados } \\
\text { à Capacidade } \\
\text { Funcional e seus } \\
\text { Determinantes }\end{array}$ & $\begin{array}{l}\text { Depressão, delirium, comprometimento cognitivo e físico (quedas, } \\
\text { redução de mobilidade), fatores ambientais (ausência de adaptações), } \\
\text { incapacidade funcional prévia e iatrogen ias (polifarmácia e } \\
\text { superproteção) }\end{array}$ \\
\hline
\end{tabular}




\section{DISCUSSÃO}

Em que pesem a relevância do tema e a consistência dos periódicos em que foram publicados os artigos, a variabilidade metodológica dificulta comparações e generalizações dos resultados.

Dentre os artigos selecionados, apenas dois foram realizados na população brasileira, o que aponta para uma carência de investigação dessa área em nosso meio..$^{21,23}$

Observou-se que, apesar de algumas semelhanças entre as amostras, o perfil sóciodemográfico da população idosa brasileira apresenta certas peculiaridades. Em concordância com a realidade mundial, no Brasil há nítido predomínio do sexo feminino e grande discrepância entre os sexos no tocante ao estado civil. No entanto, nossa população idosa, em sua maior parte, é composta por mulheres de baixa renda, analfabetas ou semianalfabetas, solteiras ou viúvas, e sem suporte familiar adequado. Além disso, embora haja um significativo aumento da população idosa acima de 75 anos, prevalecem em nosso país idosos mais jovens $-60 \mathrm{a}$ 74 anos. $^{1,3}$

Os fatores de risco citados pelos estudos, para o declínio funcional em idosos hospitalizados, puderam ser agrupados em três categorias (quadro 1): a) fatores sócio-demográficos (idade e situação de moradia/institucionalização); b) fatores médicos/clínicos (diagnóstico, múltiplas comorbidades, gravidade da condição clínica e estado nutricional); e c) fatores relacionados à capacidade funcional e seus determinantes (presença de sintomas depressivos, comprometimento cognitivo e delirium, comprometimento físico, fatores ambientais e fatores relacionados à intervenções iatrogênicas).

Quanto às características sócio-demográficas, os resultados dos estudos foram controversos. Embora a capacidade funcional seja diferente entre homens e mulheres ${ }^{28,29} \mathrm{e}$ entre pobres e ricos, ${ }^{30}$ não houve diferenças entre estes grupos em relação ao declínio funcional durante a hospitalização.

Quanto à idade, sua relação com o declínio funcional e a hospitalização não está clara. Dentre os estudos analisados, apenas um investigou a associação específica entre idade avançada e declínio funcional e demonstrou que mais de $35 \%$ dos participantes (2.761 com idade maior ou igual a 70 anos) tiveram piora em sua capacidade funcional, em relação à admissão. ${ }^{24}$ Covinsky et al. ${ }^{24}$ verificaram que os pacientes mais idosos tinham maior nível de doenças graves e de dependência funcional antes da hospitalização, quando comparados aos indivíduos idosos com menor idade da amostra. Além disso, a taxa de declínio funcional apresentou impressionante relação com a idade, excedendo $50 \%$ em pacientes acima de 85 anos. De acordo com esses autores, maiores taxas de declínio funcional e menores possibilidades de recuperação da capacidade funcional após a admissão poderiam explicar a associação entre a idade e piores resultados funcionais durante a hospitalização. 
No trabalho de Siqueira et al., ${ }^{21}$ não houve associação entre a incapacidade funcional, a faixa etária e as demais variáveis sócio-demográficas. Isso poderia ser explicado pelo elevado grau de dependência funcional (dificuldades em 7 ou mais AVDs) e de fragilidade apresentado pelos participantes mesmo antes da hospitalização. Esses resultados apoiam a tese de Ponzetto et al., ${ }^{20}$ segundo a qual a idade e outros fatores sóciodemográficos não são preditores significativos de mortalidade e resultados desfavoráveis da hospitalização, porque provavelmente outros elementos, tais como a gravidade da doença aguda à admissão, a presença de patologias crônicas e o comprometimento funcional prévio, têm maior valor preditivo do que a idade isoladamente. Além disso, em três trabalhos ${ }^{14,20,21}$ observou-se relação entre a piora clínica dos pacientes e a piora da capacidade funcional ou óbito, independentemente da idade, reforçando a importância da associação entre indicadores do estado funcional e das variáveis clínicas.

Quanto à institucionalização/situação de moradia, poucos estudos abordaram sua relação com declínio funcional. No estudo de Cornette et al., ${ }^{13}$ idosos residentes em instituição de longa permanência representavam apenas $15,7 \%$ da amostra, composta por 625 participantes, acima de 70 anos, mas foi o grupo que apresentou maior taxa de dependência funcional em AIVDs à admissão hospitalar. Dentre os $9 \%$ de idosos com comprometimento funcional da amostra total, eles representaram a maioria $-73 \%$. De acordo com comentário publicado recentemente em periódico brasileiro, idosos asilados diferenciam-se de outros pacientes hospitalizados em vários aspectos. Observase que são mais velhos, apresentam maior número de doenças e dependências físicas, psíquicas e sociais e, portanto, apresentam maior risco para declínio funcional adicional durante a permanência hospitalar. ${ }^{31}$

Apesar de citados por vários estudos, a relação entre marcadores nutricionais e inflamatórios não foi explicada com clareza. Apenas Volpato et al..$^{22}$ demonstraram, em seu estudo, que a desnutrição é uma condição comum, grave e subdiagnosticada nos idosos. História de perda de peso, sinais de desidratação, baixo índice de massa corpórea, baixa concentração de albumina e de colesterol séricos foram associados ao maior tempo de hospitalização, aumento da mortalidade e desenvolvimento de complicações como infecções e úlceras de pressão.

Quanto à condição funcional e seus determinantes, os idosos com maiores comprometimentos funcionais, déficit cognitivo e/ ou delirium à admissão hospitalar foram os que pioraram funcionalmente ou faleceram durante a hospitalização. ${ }^{19,21}$

Wakefield ${ }^{18}$ investigou a associação entre confusão aguda e resultados desfavoráveis em idosos hospitalizados e verificou que pacientes que desenvolveram confusão agu$\mathrm{da}$ (delirium) experimentaram maior declínio funcional durante a permanência hospitalar. Comparados ao grupo controle, tais sujeitos pioraram em média a dependência em duas AVDs à admissão para três AVDs 
no momento da alta. Esses resultados foram semelhantes aos encontrados no estudo de Hy Wu et al. ${ }^{27}$

Pacientes idosos com delirim tiveram alto risco para quedas e aumento da incontinência, com consequente uso de catéter urinário sem indicações clínicas, também indicado como preditor do declínio funcional por Holroyd-Leduc et al..$^{25}$ Os autores alertam que, em virtude da dificuldade no manejo desses pacientes, a equipe de saúde pode precipitar condutas iatrogênicas quanto a medicações e cuidados - restrição física, repouso prolongado no leito, polifarmácia, etc.

Alguns estudos ${ }^{18,19,21,22}$ apontam uma tendência à polifarmácia em idosos hospitalizados. Entre as medicações, o uso de ansiolíticos, antipsicóticos e antidepressivos foi considerado fator de risco potencial para o declínio funcional.

A presença de sintomas depressivos também foi frequente entre os participantes e apresentou forte associação a resultados desfavoráveis da hospitalização. ${ }^{18}$

Se a associação entre os aspectos clínicos e cognitivos e o declínio funcional está bem estabelecida, houve pouca ou nenhuma avaliação padronizada da condição motora e da mobilidade durante a hospitalização. Brown et al ${ }^{16}$ verificaram que baixos níveis de mobilidade e episódios de repouso absoluto no leito são comuns em idosos hospitalizados, ocorrendo, respectivamente, em $16 \%$ e $36 \%$ dos pacientes no estudo. Os pacientes idosos são frequentemente man- tidos em repouso no leito durante todo o período da internação, na maioria dos casos, sem justificativas por nenhum membro da equipe.

Apenas um estudo mencionou as questões ambientais, sociais e de cuidado relacionadas ao declínio funcional, durante a hospitalização. Para Kawasaki \& Diogo, ${ }^{23}$ o declínio funcional foi observado de forma mais significativa no domínio motor, quando comparado ao cognitivo/social. Segundo esses autores, as atividades avaliadas no domínio motor pela medida de independência funcional - MIF (englobando 13 itens relacionados ao autocuidado, controle de esfíncter, transferência e locomoção) integram as ações da equipe de cuidados, em especial as de enfermagem, e são por isso mais susceptíveis a iatrogenias. Quanto ao domínio cognitivo/social (englobando 5 itens relacionados à comunicação e cognição social) a memória e a interação social mantiveram valores estáveis, ao contrário da resolução de problemas, que apresentou um declínio importante, mas com melhora do desempenho, um mês após a alta.

Os mesmos autores acreditam que o declínio nas tarefas de autocuidado (banho e vestuário), no controle de esfíncteres, nas transferências e na locomoção acontece devido à inadequação estrutural e ambiental dos hospitais, como por exemplo, a ausência de barras de proteção nos banheiros, a despersonalização dos ambientes de internação e as atitudes exacerbadas de protecionismo e de amparo por parte de cuidadores e da equipe de assistência à saúde. ${ }^{28}$ 
Cinco estudos ${ }^{13,15,21,22,27}$ indicaram que pacientes idosos com tendência a quedas têm maior risco de apresentar declínio funcional durante a hospitalização. A tendência a quedas e a presença de instabilidade postural são indicadores importantes de fragilidade e podem ser resultado de: baixa visão, hipotensão postural, precário equilíbrio, nível reduzido de mobilidade, fraqueza ou doenças neurológicas. ${ }^{26,27} \mathrm{O}$ idoso frágil tem reservas fisiológicas limitadas e, quando hospitalizado, apresenta ainda maior risco de desenvolver complicações, devido ao repouso prolongado no leito e ao abandono temporário de dispositivos de auxílio, como óculos, bengalas ou andadores. ${ }^{23}$ Além disso, o descondicionamento físico, que pode começar já no segundo dia de hospitalização, torna esses pacientes vulneráveis a problemas como fraqueza, imobilidade, úlceras de pressão e infecções. ${ }^{16,27}$

Os resultados dos estudos indicaram que um número significativo de idosos acompanhados recebeu alta hospitalar com alguma forma de assistência em AVDs, principalmente as básicas, como cuidados pessoais, mobilidade e locomoção, demonstrando a importância do papel do cuidador ou familiares e a necessidade de acompanhamento mais sistematizado e rotineiro de idosos submetidos a hospitalização. 15,21,24,27

A maioria dos estudos enfocou a taxa de declínio funcional entre a admissão e a alta hospitalar, com poucos estudos descrevendo mudanças funcionais ocorridas antes e após a internação, o que exigiria maior tempo de seguimento, mas poderia fornecer um entendimento preciso das trajetórias funcionais e dos respectivos fatores de risco e consequências do declínio funcional entre idosos hospitalizados. ${ }^{18-20,23,24} \mathrm{O}$ período anterior à admissão hospitalar de duas semanas foi estabelecido como referência para definir o nível funcional prévio para a realização de AVDs e foi tomado pela maioria dos estudos, com exceção de três. ${ }^{19,20,23}$

De acordo com Covinsky et al., ${ }^{17}$ o relato retrospectivo da capacidade funcional anterior à admissão é clinicamente sensível e fornece informação prognóstica sobre a recuperação funcional e a mortalidade. Além disso, o autorrelato tanto de pacientes, quanto de cuidadores, em relação à capacidade funcional, apresentou significância estatística e foi condizente com o estado funcional na maioria dos idosos de sua amostra. No entanto, em três estudos ${ }^{14,16,19}$ a classificação do desempenho funcional de idosos feita pelos cuidadores primários, especialmente a observação realizada pela equipe de enfermagem durante a internação, foi considerada mais precisa do que o autorrelato do paciente.

Paixão Jr. \& Reichenheim ${ }^{32}$ ressaltam que os métodos habituais de se realizar uma avaliação funcional estruturada consistem na observação direta, através de testes de desempenho e utilização de questionários, quer autoaplicados ou concebidos por entrevistas face a face, entre pacientes ou cuidadores, e sistematizados por meio de uma 
série de escalas. Segundo esses autores, é a sistematização da avaliação que confere validade às informações, o que não ocorreu na maioria dos estudos analisados, que avaliaram o estado funcional por meio de medidas não padronizadas. Além disso, a capacidade funcional, dimensão-base para a avaliação geriátrica, envolve também a avaliação de fatores físicos, psicológicos, cognitivos e sociais. Essas dimensões interagem de tal forma com o estado funcional que a alteração em uma área pode indicar problemas em outras, ${ }^{32}$ o que, também não ocorreu de forma sistematizada nos estudos.

É importante salientar que não se pode perder de vista que instrumentos de avaliação padronizados, como as escalas e testes de desempenho, têm inserções e finalidades bem definidas. Apesar de serem mais acurados, sensíveis e reprodutíveis, os instrumentos não devem substituir avaliações não-padronizadas, como é o caso da anamnese e exame físico. Essas informações obtidas através de entrevista inicial e consulta de prontuários permitem maior flexibilidade e oportunidade de avaliar, de maneira mais profunda e rápi$\mathrm{da}$, respostas não esperadas, ${ }^{32}$ o que foi verificado em todos os estudos.

De um modo geral, a presença de síndromes geriátricas mostrou-se um importante determinante de resultados desfavoráveis da hospitalização, incluindo o declínio funcional. Além disso, verificou-se que complicações iatrogênicas em idosos hospitalizados são comuns e se relacionam não apenas ao uso inadequado de medicamen- tos, mas também a procedimentos diagnósticos e terapêuticos. ${ }^{15,25}$

Os dados dos estudos selecionados para esta revisão apontam para a frequência do declínio funcional na população idosa internada e endossam a necessidade de reconhecimento precoce desses indivíduos e do estabelecimento de medidas de intervenção clínicas e ambientais que possam beneficiar o idoso no período de hospitalização.

\section{CONCLUSÃO}

O declínio funcional é um fenômeno comum e esperado por um número significativo de idosos hospitalizados, em virtude da vulnerabilidade clínica, física e psicológica inerentes ao processo de envelhecimento. Ele pode ocorrer antes ou durante o período de hospitalização, e até mesmo continuar ou melhorar após a alta.

Os estudos analisados destacaram que mudanças na capacidade funcional ocorridas antes da hospitalização resultam, dentre outras causas, de doenças agudas ou da agudização de doenças crônicas e não podem ser prevenidas exclusivamente pelo cuidado fornecido no hospital. Ao contrário, o comprometimento funcional ocorrido após a admissão reflete a interação da condição clínica com o tratamento hospitalar disponibilizado e é passível de ser amenizado.

A presença de déficit cognitivo, de incapacidade prévia em AVDs, de histórico de 
quedas, de baixo nível de mobilidade e de instabilidade postural, além de fatores ambientais, foram citados como fatores predisponentes do declínio funcional.

A partir da análise crítica dos estudos, pode-se destacar que é de extrema importância o conhecimento sobre a condição clínica, o nível físico, cognitivo e funcional do idoso prévio à internação, para que se possam estabelecer objetivos realistas e eficazes, minimizando ou controlando os fatores de risco para o declínio funcional durante ou após o período de hospitalização.

Cabe ressaltar a necessidade de mais estudos, especialmente na população brasileira, em que os hospitais estão despreparados para cuidar da população idosa; equipes

\section{REFERÊNCIAS}

1. Camarano AA. Envelhecimento da população brasileira: uma contribuição demográfica. In: Freitas EV et al. Tratado de Geriatria e Gerontologia. Rio de Janeiro: Guanabara Koogan; 2006.

2. Costa AJL. Metodologias e indicadores para a avaliação da capacidade funcional: análise preliminar do Suplemento Saúde da pesquisa Nacional por Amostra de Domicílios - PNAD, Brasil, 2003. Cien Saude Colet 2006; 11(4): 927-40.

3. Paschoal SMP; Franco RP; Salles RFN. Epidemiologia do envelhecimento. In: Papaléo Neto M. Tratado de Gerontologia. 2.ed. São Paulo: Atheneu; 2007.

4. Chaimowicz FA. Saúde dos idosos brasileiros às vésperas do século XXI: multidisciplinares de cuidado, com foco em reabilitação, ainda não são a regra, e estudos relacionados a este fenômeno ainda são escassos.

Por fim, a partir dos fatores de riscos identificados pelos estudos, sugerem-se a validação de instrumentos de avaliação padronizados e adequados ao perfil demográfico, funcional e epidemiológico dos idosos brasileiros hospitalizados, assim como a realização de ensaios clínicos randomizados para se investigar e estabelecer estratégias de intervenção para a prevenção do declínio funcional nesta população, haja vista que, dependendo da trajetória do declínio, os fatores citados nesta revisão podem funcionar ora como fatores de risco, ora como consequência do declínio funcional.

problemas, projeções e alternativas. Rev Saúde Pública 1997; 31: 184-200.

5. Organização Mundial de Saúde. CIF: Classificação internacional de funcionalidade, incapacidade e saúde. [ tradução do centro colaborador da OMS para a classificação de Doenças em Português]. São Paulo: Edusp, 2003.

6. Farias N; Buchalla CM. A Classificação internacional de funcionalidade, incapacidade e saúde: conceitos, usos e perspectivas. Revista Brasileira de Epidemiologia 2005; 8(2): 187-93.

7. Coelho Filho JM. Modelos de serviços hospitalares para casos agudos em idosos. Rev Saúde Pública 2000; 34(6): 666-71. 
8. Creditor MC. Hazards of hospitalization of the elderly. Ann Intern Med February 25, 2008; 168(4): $390-6$.

9. King BD. Functional decline in hospitalized elders. Medsurg Nurs Oct 2006; 15(5): 265-71.

10. Hoenig HM, Rubenstein LZ. Hospitalassociate desconditioning and dysfunction. J Am Geriatr Soc 1991; 39: 220-2.

11. Sager MA, et al. Hospital admission risk profile (HARP): identifying older patients at risk for functional decline following acute medical illness and hospitalization. J Am Geriatr Soc 1996; 44(3): 251-7.

12. Inouye $\mathrm{SK}$, et al. Importance of functional measures in predicting mortality among older hospitalized patients. JAMA 1998; 279: 1187-93.

13. Cornette P, et al. Early evaluation of the risk of functional decline following hospitalization of older patients: development of predictive tool. Eur J Public Health 2005 sep; 16(2): 203-8.

14. Espaulella J, et al. Time-dependent prognostic factors of 6-month mortality in frail elderly patients admitted to postacute care. Trajectories of functional and nutricional status and mortality. Age and Aging july 1, 2007; 36(4): 407 - 13.

15. Anpalahan M, Gibson SJ. Geriatric syndromes as predictors of adverse outcomes of hospitalization. Intern Med J 2008 jan;38(1):16 - 23.

16. Brown CJ, Friedkin RJ, Inouye SK. Prevalence and outcomes of low mobility in hospitalized older patients. J Am Geriatr Soc 2004 aug; 52(8): 1263 -70.

17. Covinsky KE, et al. Functional status before hospitalization in acutely ill older adults: validity and clinical importance of retrospective reports. J Am Geriatr Soc 2000 feb; 48(2): 164-9.

18. Wakefield BJ. Behaviors and outcomes of acute confusion in hospitalized patients. Appl Nurs Res 2002 nov; 15(4): 209-16.

19. Wakefield BJ, Holman JE. Functional trajectories associated with hospitalization in older adults. Western Journal of Nursing Research 2007 mar; 29(2): 161-77.

20. Ponzetto M, et al. Risk factors for early and late mortality in hospitalized older patients: the continuing importance of functional status. Journal of Gerontology: Medical Sciences 2003; 58(11): 1049-54.

21. Siqueira $A B$, et al. Impacto funcional da internação hospitalar de pacientes idosos. Rev Saúde Pública 2004; 38(5): 687-94.

22. Volpato S, Onder G, Cavalieri M. Characteristics of nondisabled older patients developing new disability associated with medical illness and hospitalization. Journal General Internal Medicine 2007 mar; 22(5): 668-74.

23. Kawasaki K, Diogo MJD'E. Impacto da hospitalização na independência funcional do idoso em tratamento clínico. Acta Fisiátrica 2005; 12(2): 55-60.

24. Covinsky KE; et al. Loss of independence in activities of daily living in older adults hospitalized with medical illness: increased vulnerability with age. J Am Geriatr Soc 2003 apr; 51(4): 451-8.

25. Paixão Jr CM; Reichenheim ME. Uma revisão sobre instrumentos de avaliação do estado funcional. Cad Saúde Pública 2005; Jan-Fev; 21(1): 7-19.

26. Holroyd-Leduc JM, et al. The relationship of indwelling urinary catheters to death, length of hospital stay, functional decline and nursing home admission in 
hospitalized older medical patients. Journal of American Geriatrics Society 2007 Feb; 55(2): 227-33.

27. Lindenberger EC, et al. Unsteadiness reported by older hospitalized patients predicts functional decline. Journal American Geriatric Society 2003 may, 51(5): 621-6.

28. Hy Wu MBBS, Sahadevan S, Ding YY. Factors associated with functional decline of older persons following discharge from an acute geriatric unit. Annals Academy of Medicine Singapore 2006 Jan; 35(1): $17-23$.

29. Parahyba MI; Simões CCS. A prevalência de incapacidade funcional em idosos no Brasil. Cien Saude Colet 2006; 11(4): 967-74.

Recebido:19/11/2008

Aprovado:5/6/2009
30. Stuck AE, et al. Risk factors for functional status decline in community-living elderly people: a systematic literature review. Social Science \& Medicine 1999 48: 445-69.

31. Lima-Costa MF, Matos DL, Camarano AA. Evolução das desigualdades sociais em saúde entre idosos e adultos brasileiros: um estudo baseado na Pesquisa Nacional por Amostra de Domicílios (PNAD 1998, 2003). Ciência e Saúde Coletiva 2006; 11(4): 941-50.

32. Gorzoni ML, Pires SL. Idosos asilados em hospitais gerais. Rev Saúde Pública 2006; 40(6): 1124-30.

33. Paixão Junior CM; Reichenheim ME. Uma revisão sobre instrumentos de avaliação do estado funcional. Cad Saúde Pública 2005 Jan-Fev; 21(1): 7-19. 